\title{
Feature-based geometric reasoning for process planning
}

\author{
G ADITYA NARAYAN, S R P RAO NALLURI and B GURUMOORTHY \\ Department of Mechanical Engineering, Indian Institute of Science, Bangalore \\ 560012 , India \\ e-mail:bgm@mecheng.iisc.ernet.in
}

\begin{abstract}
We present a framework based on Domain Independent Form (DIF) features for automatic evaluation of manufacturability and process planning for machining. The framework enables interpretation of a common product model with respect to each task in the transition from design to manufacture. A key idea here is to generate the interpretation suitable for each task in two steps. In the first step, DIF features that are defined through feature enumeration are automatically extracted from the geometric model. The extracted DIF features are then mapped into features meaningful for individual tasks through geometric reasoning based on domain dependent knowledge. The formal approach to feature definitions and separation of the domain specific reasoning from the general geometric reasoning enable us to overcome the bottlenecks reported in features technology.
\end{abstract}

Keywords. Feature-based manufacturing; feature extraction; feature mapping; visibility; process planning; manufacturability evaluation.

\section{Introduction}

The concept of feature evolved from the desire to devise methods for integration of part geometric model with applications such as process planning, group technology coding and numerically controlled (NC) tool path programming. Features are high level abstractions of part geometry such as holes, slots and ribs around which engineering knowledge and expertise is structured. Hence reasoning about part geometry in applications such as process planning becomes easier to automate when part description becomes available in terms of features rather than basic topological entities (face, edge and vertex) that are available in the geometric model. These features are referred to as form features as they describe the form and shape of a part.

The motivation for integrating part geometric model (also referred to as the CAD model) with applications such as planning for manufacture, comes from the need to compress product development lead times and to reduce costs. The sequential nature of product design and the necessity of human intervention in the transition between individual tasks have been responsible for large lead times and costs in the conventional product development 
cycle (Bakerjian 1992). By allowing downstream lifecycle concerns to influence product design during the design process itself, delays and costs due to the sequential nature of the development process can be reduced considerably, if not eliminated. In the context of products manufactured by machining, this would imply ensuring that the design is machinable during the design process itself. Eliminating human intervention in the transition between tasks involves automating the geometric reasoning involved in interpreting part geometry appropriate to the task domain and the process-based reasoning to realise the task.

Automation of evaluation of manufacturability and planning of manufacture requires that part information be available at different levels of abstraction and detail. For instance the task of generating a process plan involves sequencing of machining instructions (gross process plan) and generation of NC tool path (fine process plan). The first requires information on entities such as slot, step or hole, whilst the second also needs the geometric details such as surface normals and locations. The domain knowledge/expertise of each task in product development is structured around features. Hence it is essential to support features to automate the reasoning involved in evaluation of a design with respect to a task.

There have been three routes followed by researchers to realize feature information to reason about process (Shah 1991). These are as below.

- Interactive feature definition: In this approach, features are defined by the users by picking entities, associated with each feature, from a CAD model. This approach has been used mainly to input data to process planning and tool path generation programs (Chang \& Wysk 1985).

- Feature-based modelling (FBM): In this approach the feature information is incorporated in the model construction stage itself. This is achieved by using features to build the model (Salomons et al 1993). This method of modelling is a generalisation of the 'design by features' approach. In the 'design by features' approach only design features are used, whereas in FBM, features of interest to any one specific application can be used. In most implementations, feature-based modelling is based on Constructive Solid Geometry (CSG), where the features are available as primitives in addition to the regular primitives such as cylinder and block. Commands of a solid modeller to create the feature are generated based on the feature selected. Arbab (1992) modelled parts by removing volumes corresponding to machining features from the stock solid. Part feature model thus obtained was used to link design with process planning. Shah (1988) implemented a general purpose feature-based modelling system. This system allows the user to define a set of features along with all its manipulation functions.

- Automatic feature extraction: In this approach, form features of interest to an application are automatically extracted from the geometric model of a part. Automatic feature extraction has fundamental significance from the integration perspective of product development cycle. Reasoning involved in the downstream applications cannot be automated without correct interpretation of part design with respect to each task. Since most of the established application knoweldge is based on human interpretation of part geometry, it is essential to generate human-like interpretation for complete automation.

Automatic extraction of features from a geometric model is a difficult problem. Application and context dependence of features make it doubly difficult. The basic process involves matching feature definitions with regions of geometric model. Several approaches 
have been proposed to solve this problem. These vary from syntactic pattern recognition techniques (Kyprianou 1980), graph matching (Joshi \& Chang 1988), and volumedecomposition (Shah et al 1994; Tseng \& Joshi 1994; Sakurai \& Dave 1996) to hint-based techniques (Vandenbrande \& Requicha 1993). The reader is referred to Shah (1991) and Subrahmanyam \& Wozny (1995) for reviews of the literature on feature recognition.

None of the commercial CAD/CAM/CAE software that claim to be feature-based can integrate design with manufacturing, analysis with other downstream tasks. Most of these software support only the FBM approach. In this approach a task-specific model can be obtained only by restricting the user to models using a particular set of features. For other tasks, the part has to be modelled differently and hence both data integrity and development time are adversely affected.

On the other hand, feature recognition approach to generate feature models is only now beginning to see commercial development (CAD 1997). Most of the techniques reported in literature have limited scope and their extension to handle real world part complexity is not proven. Implementations reported so far consider only one application (process planning for machining) and domain specific heuristics are used to recognise interacting features (Subrahmanyam \& Wozny 1995). The most formal and general approach is graph matching, but sub-graph isomorphism is in NP-complete list. This approach also suffers from combinatorial explosion problem as sub-graphs of all feature instances have to be defined in advance and checked for. Techniques based on decomposition (Shah et al 1994; Tseng \& Joshi 1994; Sakurai \& Dave 1996) are proving to be more successful than graphbased approaches in handling feature interactions, at least for simple cases.

In the proposed framework, domain-independent form (DIF) features are used as the intermediate representation. The term 'domain-independent' is used to imply that the definitions and classification of the features are based purely on form (geometry and topology) and not on considerations arising from various application domains such as machining, near-net shape processing, analysis, or assembly. The DIF feature set is then used to obtain a feature set corresponding to other application domains through a process called feature mapping. In the following we first present the feature-based framework for manufacturing (in particular, machining). Definitions for DIF features are presented next. We then highlight some of the results in feature extraction, mapping and manufacturability evaluation. We conclude with some open issues in this area.

\section{Feature-based manufacturing framework}

A product typically consists of several electrical, electronic and mechanical parts. The framework proposed addresses only the development of discrete mechanical parts. Part development involves more than one task, and even in a single task part data may have to be interpreted in more than one way. For example, if a part has to be manufactured through machining, then it has to be considered for process planning, which in turn consists of several sub-tasks such as stock selection, fixturing, sequencing, tool selection, NC tool path generation and final inspection.

Generating part interpretations suitable to each task through FBM approach would involve modelling the same part differently for each task. This would adversely affect the 


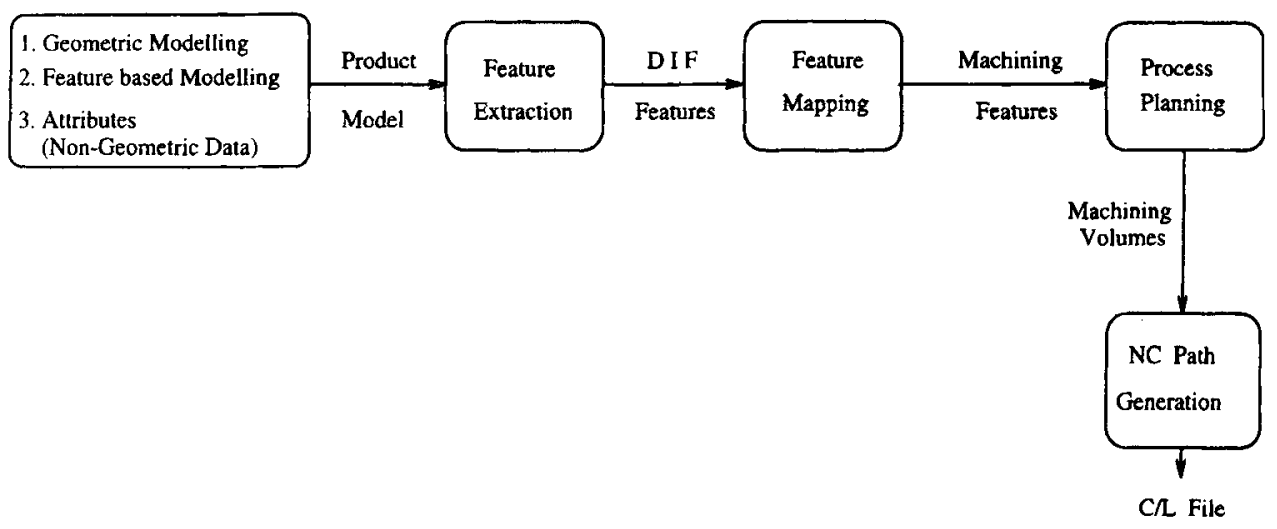

Figure 1. Feature-based machining system.

concurrency and development time. On the other hand, implementing feature extraction specific to each application involves bringing down the level of abstraction of each application to the level of basic geometry, which is a difficult task. Moreover, automatic handling of non-geometric data which can only be attributed to features is not clear at present in the feature recognition approach.

The proposed framework is designed such that feature-based modelling (FBM) and feature extraction approaches complement each other (see figure 1). Migration from geometric modelling to product modelling is effected through providing features for modelling. Product data which are difficult to attribute to basic geometric entities can be attributed to features. Unlike the conventional FBM approach, the designer is not restricted to use only features for modelling and can use boolean operations over primitives along with features. As features get modified in the process of modelling, the design model does not correspond to the feature model of the final solid. In the proposed approach, part geometry is represented as boundary representation (B-rep). Product data are represented as attributes to entities in the design model and geometric model.

Design model is an unevaluated representation of the design process. Reasoning over design model to generate task-specific interpretations is difficult because of its subjectivity (a designer's viewpoint) and non-uniqueness. Hence the design model is referred only for product data in the mapping process. From the evaluated geometric model (B-rep), DIF feature model is created through automatic feature extraction. Then the extracted DIF features are mapped into each specific task. DIF feature interpretation is at a higher level of abstraction than the basic geometry. Hence, knowledge-based reasoning involved in mapping can be implemented without bringing down the domain knowledge of each task to the level of basic geometry.

We have taken a general and formal approach to DIF feature classification and definitions. Based on this formalisation, problems in feature recognition and feature-based modelling are treated generally without using any one domain specific heuristic. All the task specific issues are addressed in feature mapping and subsequent process specific modules, process planning and $\mathrm{NC}$ tool path generation (figure 1), where domain knowledge interacts with the part's DIF interpretation. Process-based reasoning has been divided into three modules, as the interaction of process and part geometry is of different degrees in 


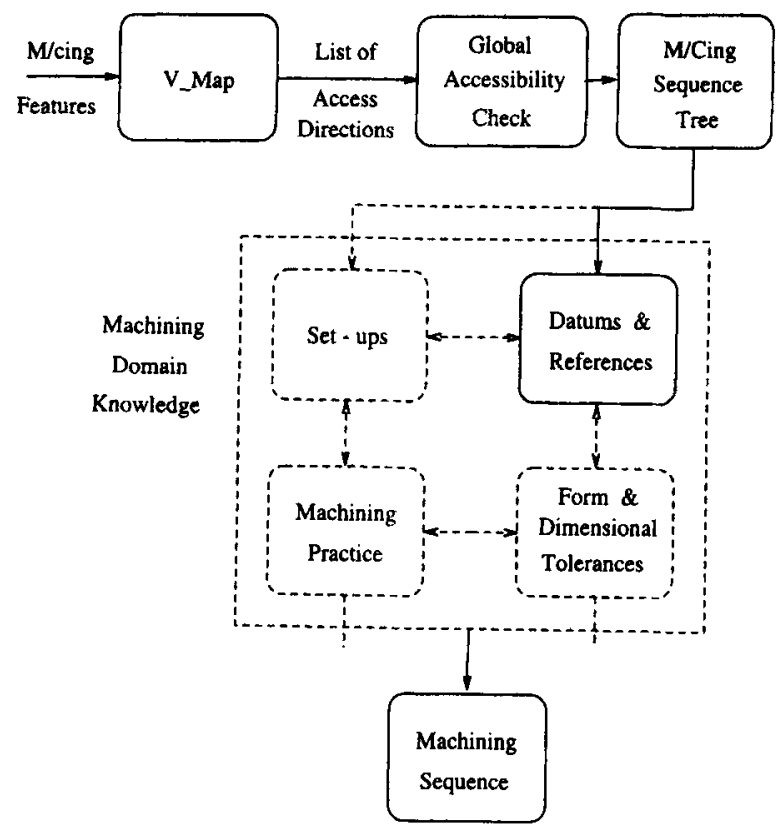

Figure 2. Manufacturability evaluation and planning for machining.

each of the modules and the nature of reasoning is also different. For example, in NC tool path generation the task primarily involves geometric computations, once the cutting parameters are fixed in the planning module.

In this paper, we present a feature-based approach for the planning module where the objective has been to use geometric reasoning to provide plans that can then be pruned by constraints from the process or processing environment. This is illustrated in the schematic of figure 2. The output from the mapping module of figure 1, machining features, forms the input to this module. Module V_Map uses visibility checks on the faces of each feature to generate access directions for machining the feature. A global accessibility check along all/feasible set of the access directions is then used to evaluate manufacturability of the part and if the part is manufacturable, to generate all possible sequences for machining the part. This sequence tree is then pruned by subjecting it to constraints from the machining domain such as set-up optimisation, handling datums/references, existing machining practice to obtain one or more machining sequence. The remainder of the paper explains this approach and provides some results.

\section{Domain-independent form features}

Both feature extraction and feature-based modelling require a set of well-defined form features. A feature has to be defined either interactively or through a language before it can be used either by feature recognition or by feature-based modelling modules. Form features unique to certain applications such as twists, louvres, tabs of sheet metal, and secondary features such as knurl, teeth and threads interact with the existing part geometry in a fixed way. It is the primary features that have an associated volume whose interpretation 

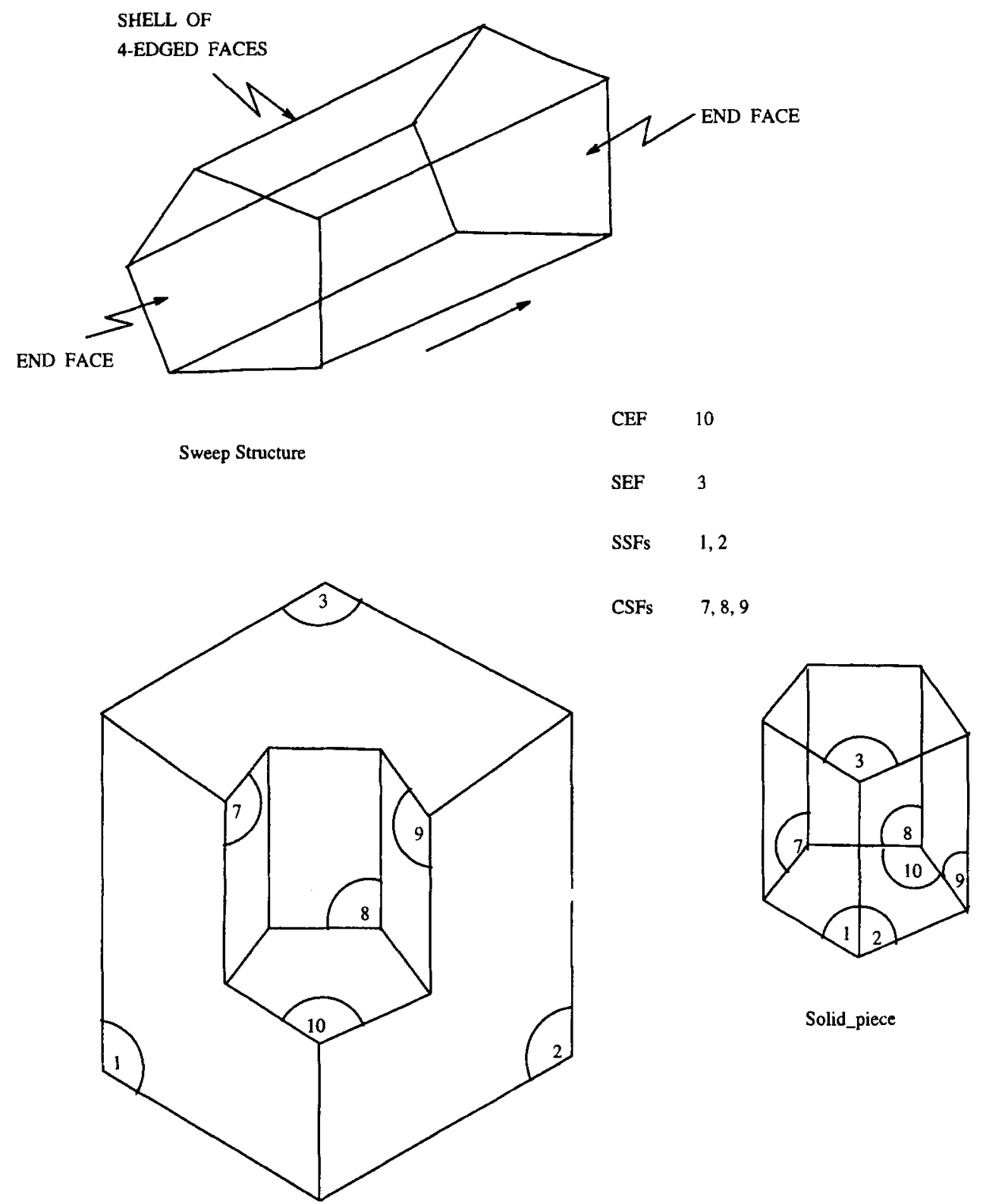

Figure 3. Sweep structure and face classification for DIF features.

depends on the application, context, and interactions. Hence we focus on classification, extraction, mapping and manipulation of primary (non-unique) features.

The conventional approach (Butterfield et al 1986) is first to collect form features of interest to a particular application. Collected features are then organised through classification into hierarchies and feature definitions are derived. In this approach, the number of form features collected and the criteria used for classification are subjective. Hence finiteness and completeness of the defined feature set cannot be established with respect to possible variation of topology and geometry in designed components. Unlike earlier 
Table 1. Basic DIF feature types.

\begin{tabular}{|c|c|c|c|c|}
\hline \multirow[b]{2}{*}{$\begin{array}{l}\text { No. of } \\
\text { shell faces }\end{array}$} & \multicolumn{4}{|c|}{ No. of end faces } \\
\hline & $\begin{array}{l}\text { No ends } \\
\text { (closed) }\end{array}$ & $\begin{array}{c}0 \\
\text { (Double blind) }\end{array}$ & $\begin{array}{c}1 \\
\text { (Blind) }\end{array}$ & $\begin{array}{c}2 \\
\text { (Through) }\end{array}$ \\
\hline $\begin{array}{l}0 \\
\text { (Holes) }\end{array}$ & $\begin{array}{c}\text { Closed } \\
\text { hole }\end{array}$ & $\begin{array}{c}\text { Double } \\
\text { blind hole }\end{array}$ & $\begin{array}{l}\text { Blind } \\
\text { hole }\end{array}$ & $\begin{array}{l}\text { Through } \\
\text { hole }\end{array}$ \\
\hline $\begin{array}{l}0 \\
\text { (Slots) }\end{array}$ & $\begin{array}{l}\text { Closed } \\
\text { slot }\end{array}$ & $\begin{array}{l}\text { Double } \\
\text { blind slot }\end{array}$ & $\begin{array}{l}\text { Blind } \\
\text { slot }\end{array}$ & $\begin{array}{l}\text { Through } \\
\text { slot }\end{array}$ \\
\hline $\begin{array}{l}2 \text { Adjacent } \\
\text { (corner slots) }\end{array}$ & $\begin{array}{l}\text { Closed } \\
\text { corner slot }\end{array}$ & $\begin{array}{l}\text { Double blind } \\
\text { corner slot }\end{array}$ & $\begin{array}{l}\text { Blind } \\
\text { corner slot }\end{array}$ & $\begin{array}{l}\text { Through } \\
\text { corner slot }\end{array}$ \\
\hline $\begin{array}{l}2 \text { Non-adjacent } \\
\text { (virtual slots) }\end{array}$ & & $\begin{array}{l}\text { Double blind } \\
\text { virtual slot }\end{array}$ & $\begin{array}{c}\text { Blind } \\
\text { virtual slot }\end{array}$ & $\begin{array}{l}\text { Through } \\
\text { virtual slot }\end{array}$ \\
\hline $\begin{array}{l}\text { Three or more than } \\
3 \text { adjacent } \\
\text { (virtual corner slots) }\end{array}$ & & $\begin{array}{l}\text { Double blind } \\
\text { virtual corner slot }\end{array}$ & $\begin{array}{l}\text { Blind } \\
\text { virtual corner slot }\end{array}$ & \\
\hline
\end{tabular}

approaches we have defined features through enumeration of possible topological variations in designed solids.

We have modelled form feature-generation as subtraction/addition of a solid_piece from/to a base_solid (Rao Nalluri 1994). The solid on which the feature is created is referred as the base_solid and the minimum solid that is required to create the feature is referred as the solid_piece. In the generation of a feature, some existing faces of the base_solid are modified and some new faces are created. The modified and the newly created faces are classified as 'shared' and 'created' faces respectively w.r.t. the generated feature. The solid_piece faces can be classified into 'shell' and 'end' faces, and combining the two classifications results in four face types: Created Shell Face (CSF), Created End Face (CEF), Shared Shell Face (SSF) and Shared End Face (SEF) as shown in figure 3. The geometry and arrangement of 'created' faces of the feature is defined as the 'shape' of the feature. The connectivity of the feature face set with the rest of the base_solid is defined as 'type' of the feature. In the feature generation model, keeping the variations which affect the 'shape' independent, variations which affect 'type' are enumerated and generic feature definitions are derived in terms of the four face types as given in table 1.

\section{Direct manipulation through form features}

Feature-based modelling (FBM) provides an intuitive design environment and enables attribution of product data to the features. The common approach to implement FBM system is to have features supported only at the user interface. For all manipulations of geometric representation, features are converted into solid primitives and boolean operations. In contrast to earlier approaches we have developed algorithms for direct manipulation (Rao Nalluri \& Gurumoorthy 1993a) of boundary representation (B-rep) through features. Feature generic 'type' dictates the manipulation involved in creation and deletion of a feature instance. All feature-based editing of B-rep are effected through deletion of the old feature and creation of a modified feature. Feature relations are captured through 
shared/created face classification. Automatic adjustment of features in editing is effected based on the captured feature relations. Manipulation of B-rep directly with features through computationally efficient incremental upgradation distinguishes our work from other efforts in this area.

\section{Extraction of DIF features}

Extraction of DIF features involves decomposing of the input solid into a set of instances of enumerated generic form features. We have modelled the feature extraction process as a reverse process of feature generation. In the process of feature generation the topological complexity of the base_solid increases. Hence in the process of feature extraction part solid should get simplified with extraction of each feature. The idea of topological simplicity is implemented through the concept of Dynamic Topological Status (DTS) (Rao Nalluri 1994; Hari 1995). DTS of a face is a set of topological factors about the face which describe its topology. Since features are created in a certain order there is an order in their extraction. A feature gets extracted when all its faces are only 'created' faces and not the 'shared' faces of any other features. 'Created' faces have simple topology compared to 'shared' faces. DTS values of faces enable clustering of 'created' faces of a feature and also give clues about the probable 'type' of the feature. The probable feature 'type' is confirmed based on the enumerated feature definitions. For example, the clue for a blind_slot feature is a face with all 4-edged neighbours except one corresponding to single shared shell face (SSF). The confirmation procedure for feature type blind_slot is as given below. Once the 'type' is fixed, the feature face set is identified and fixing 'shape' becomes a local matching problem.

Procedure. Blind-slots(face[i])

Feature-Type $:=$ NULL

If (face[i] DTS $1 !=4$ and DTS2 $=1$ and DTS3 $=0$ )

End-face $1:=$ face[i]

Find the single non-four-edged neighbor of End-face1

If (It is of type SSF)

Find End-face2

IF(DTS of End-face1 and End-face2 are IDENTICAL)

IF(Angle btw End-facel and CSF's is CONVEX)

Feature-Type:= Double Blind Slot

ELSE

Feature-Type:= Double Blind Rib

ELSE

IF(Angle btw End-facel and CSF's is CONVEX)

Feature-Type:= Blind Slot

ELSE

Feature-Type:= Blind Rib

\section{End Procedure}

Return(Feature-Type) 
The separation of 'type' and 'shape' in feature definitions reduced the number of confirmation procedures required from $\left(n_{t} \times n_{s}\right)\left(n_{t}=\right.$ number of types, $n_{s}=$ number of shapes) to $\left(n_{t}+n_{s}\right)$. The complexity of the search depends on the number of faces rather than the number of feature 'types' supported. Hence the complexity of feature extraction is $O\left(n_{f}{ }^{2} \log \left(n_{f}\right)\right)$. Since all feature interactions are covered in enumeration for defining features, interactions do not require any special heuristic procedures for their resolution. The order obtained in the extraction can be used in mapping.

Inability to deal with interacting and intersecting features in a general way is one of the bottlenecks in features technology. We attribute this problem to (1) incompleteness of the feature set w.r.t. to topological and geometric variation in parts, (2) attempting to recognise a task specific feature when it is not explicit through relaxation of feature definition and application of domain-specific heuristics. The formal approach to feature definitions enabled us to define a complete set of feature types that is required to cover the defined topological and geometric variation. In the case of intersecting features, as we do not attempt to recognise the task-specific features directly, the most explicit feature is recognised without any application concern. Later in the mapping, based on the relationships among extracted DIF features, application specific interpretation is obtained.

\section{Mapping DIF features to applications}

Mapping involves knowledge-based reasoning along with geometric reasoning. Relations among extracted DIF features are explicitly represented as Feature Relational Graph (FRG) to apply the high level application knowledge. FRG is constructed based on the shared/created face classification. A feature $\mathrm{X}$ is said to be a child of another feature $\mathrm{Y}$, if and only if, at least one shared face of feature $X$ corresponds to a created face of feature $Y$. FRG is an acyclic graph which stores the child/parent relationships among features. Mapping modules have access to (1) geometric model, (2) FRG of extracted DIF features, and (3) design model. Both (1) and (2) representations are domain-independent, and entities in representation and their interpretation are well defined, whereas the design model is a designers interpretation and hence is subjective. It is mainly referred to get the task-specific product data. These three interpretations at different levels of abstraction provide all the information at the required levels to enable knowledge-based reasoning in mapping.

The inputs to the mapping module are stock B-rep, part B-rep, and FRG. The feature extraction module classifies the DIF features extracted based on shape (rectangular, circular, I-section etc.), class (hole, slot, step) and type (positive, negative). With reference to the feature definition, positive features and negative features are obtained by adding and removing material respectively from the base_solid. Negative features without positive children map directly into machining domain. Positive features that are child features of a negative feature are split into the portion totally inside the negative feature volume and the portion outside. The portion outside the negative feature volume is treated as a separate positive feature then onwards. The portion of the positive child feature inside the negative feature and the negative feature are combined as a single negative machining zone. Positive features which are adjacent are clustered and enclosed with a box. Positive feature cluster and box together form a positive machining zone. The boxes corresponding to positive 


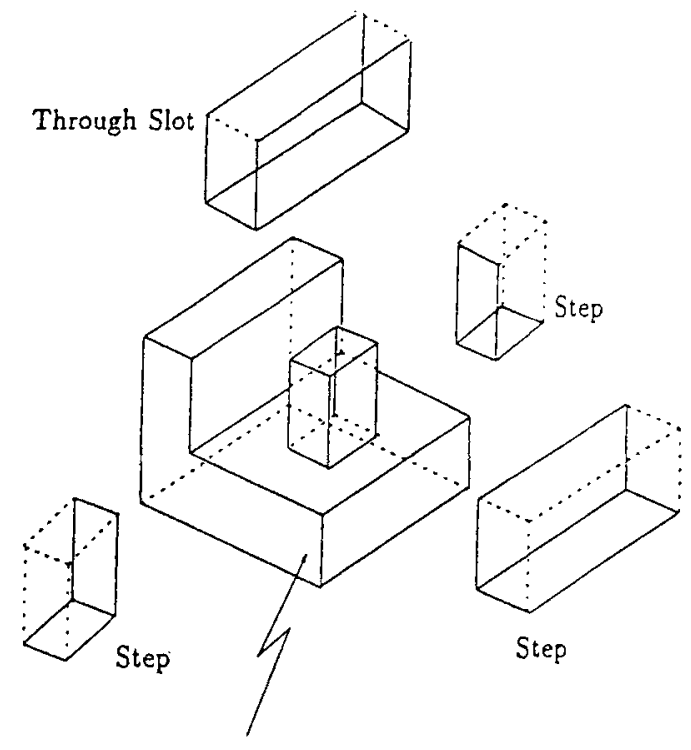

Part with Positive
Figure 4. Mapping DIF features to machining features.

machining zones and base_solid make the first stage solid. First, stock solid is reduced to first stage solid through clipping (Rao Nalluri \& Gurumoorthy 1993b; Rao Nalluri 1994), then enclosing box in each positive machining zone is clipped w.r.t. inside feature cluster. In clipping a outside solid w.r.t. an inside solid, the outside solid is split about the faces of the inside solid. The results of clipping are convex volumes that are required to be subtracted from the outside solid to get the inside solid. After the clipping, all positive features and the base_solid are created in the stock solid. As all negative features have to be either on base_solid or on one of the positive features, they can be machined any time after their shared faces are ready. Through clipping all part positive features are converted into negative machining features w.r.t. stock. These negative features are added to the part features list for further processing. Figure 4 shows a part with positive DIF feature and the negative features obtained after mapping.

\section{Manufacturability evaluation}

Features extracted from the B-rep of the solid or obtained through the mapping process described above are regions determined only by local topological and geometric characteristics of the part. A visibility-based approach has been developed to find the global attributes (accessibility) of features and determine how this affects the manufacturability of the part. An added gain from this approach has been the usefulness of the global attributes in process planning. Local accessibility of a feature is first determined based on the visibility of faces forming the feature and machining access directions are generated. The global accessibility of each feature along the machining directions is then assessed with respect to the part (Aditya Narayan 1995). Based on the global accessibility information it is possible to enumerate the machining sequences that will realise the part from stock. This enumeration can then be pruned using other constraints such as dimensional 

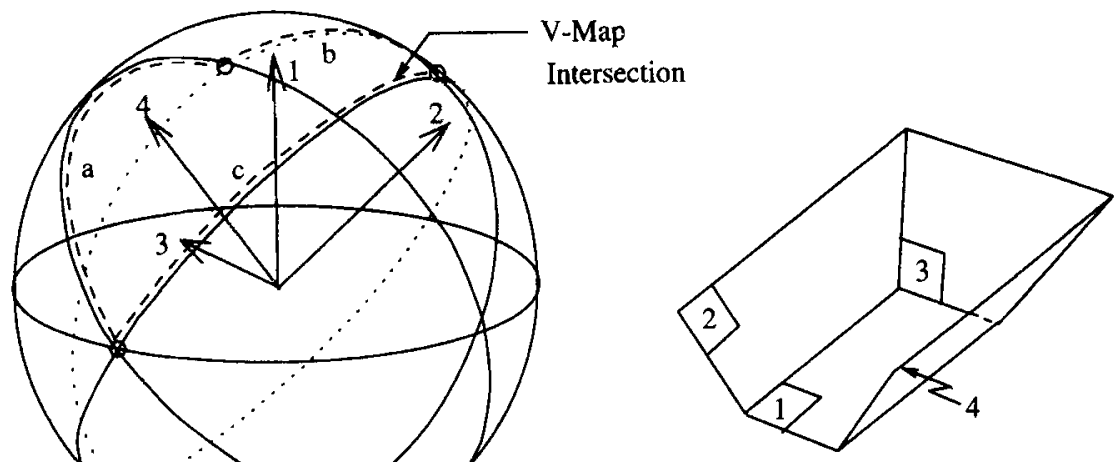

Feature CSFs 1, 2, 3, 4

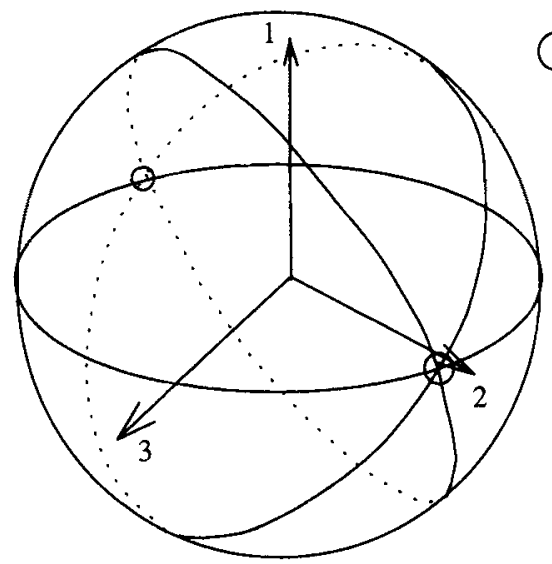

V-Map
Intersection

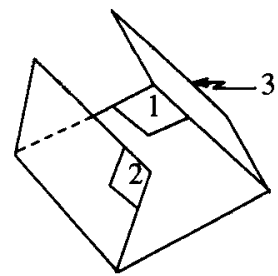

Feature CSFs: $1,2,3$

Figure 5. Visibility map (V_Map) of features.

data/references, set-up minimisation, tool availability etc. to generate machining process plans.

Local accessibility of a feature refers to the existence of directions from which all points of interest on the feature are visible. In the context of feature-based manufacturing, the objective is to find the directions from which all the faces of a feature are completely visible. These are directions in which a tool might be able to approach a feature.

Visibility maps (V_Maps) (Woo \& Chen 1992; Woo 1994) can be used to determine the local accessibility of a feature. A feature is said to be locally accessible if there is a direction in which all the created faces of a feature are visible. As a point in the V_Map of a surface denotes a direction from which all the points on the surface are visible, for all the created faces of the feature to be visible, the V_Maps of the created faces must intersect. The valid directions of access for the feature will lie within this intersection of face V_Maps. This region of intersection of the V_Maps of the feature's created faces is 
called the V_Map of the feature. Figure 5 shows the V_Map of two features. For the V-slot shown on top, the V_Map is a finite region (indicated by the dotted line in figure 5). This implies that there are many access directions for the feature. For the dovetail slot (bottom of figure 5) the intersection of the V.Maps of the created faces are just two points that are shown circled in the figure. The dovetail slot, therefore can be accessed for machining only from the two directions that are normal to the two end faces.

Valid access directions are those which map to a point on the unit sphere that is above or on the planes corresponding to the created faces of the feature. If no V_Map can be generated for a feature, the feature is not locally accessible. Such features are to be handled either by changing the design of the feature to make them locally accessible or by resorting to special manufacturing methods, such as the use of special tools. Parts with such features are not completely manufacturable. At present such cases of local inaccessibility are flagged to the user.

The feature V_Map determines the local accessibility of features and is a characteristic of the feature only. They are sensitive only to orientation of the feature and not to its position in space. The feature V_Map is a semi-infinite set of directions, from which a finite set of directions has to be picked for evaluating global accessibility of the feature along these directions. In actual machining practice, the choice of a particular access direction is based on heuristics. To generate as many relevant access directions as possible, candidate directions, based on some common cases encountered in general machining, are tested. Thus, normals to the created (CEFs and CSFs) and the shared faces (SEFs and SSFs) are checked with the feature V.Map and are taken as valid access directions if they lie within it. These directions form the initial set of access directions which will be checked for global accessibility of features.

\subsection{Global accessibility}

Determination of global accessibility is a check of whether a generic tool, representable as a line, can reach the feature to machine it. Global accessibility of a feature does not assure absolute manufacturability of the feature. It is only a first-step evaluation before a more detailed tool and manufacturing environment specific accessibility analysis (that includes size effects) is done.

The traditional method of determining global accessibility by ray tracing from the region whose global accessibility is to be determined (Spyridi \& Requicha 1990; Vandenbrande \& Requicha 1993; Lim \& Menq 1994) is a computationally intensive task if carried out for the whole feature. Further, ray tracing is susceptible to failure due to bad choice of ray spacing and poor resolution. We define a feature to be globally accessible, along a direction, if the intersection of the part or workpiece and the extrusion of the feature volume in that direction is NULL i.e.

$$
\left(i W \cap \operatorname{Ext}\left(V_{F}\right)_{l}\right)=\Phi
$$

where $i W=$ interior of workpiece and $\operatorname{Ext}\left(V_{F}\right)_{l}=$ extrusion to infinity of feature volume, $V_{F}$ along a direction $l$ (see figure 6). Mill et al (1994) also use a volume-based approach but their method does not yield correct results for directions of access that are not normal to the assumed feature-machining face or for features such as dovetail slots. 

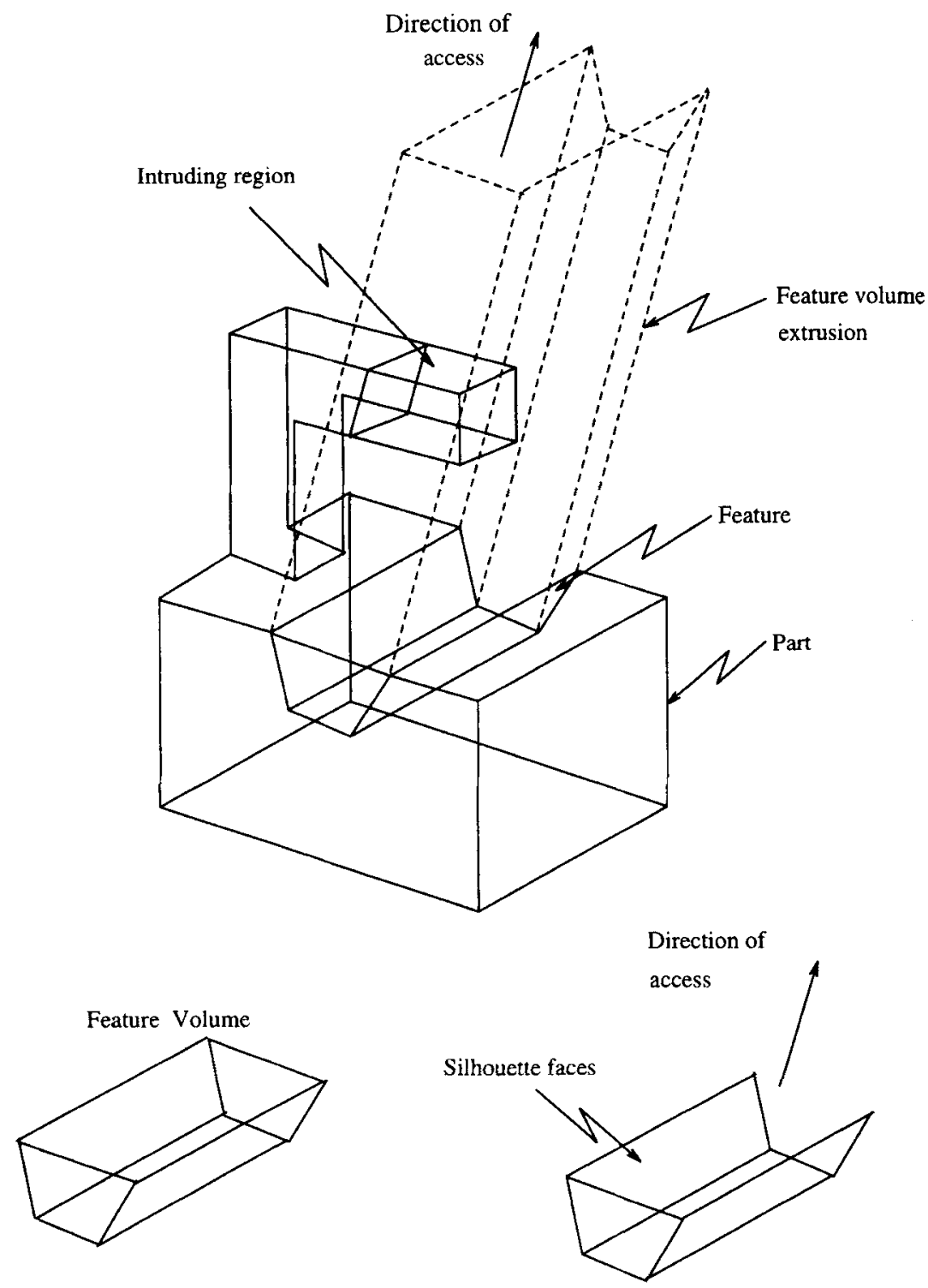

Figure 6. Determination of global accessibility of a feature.

The premise for using the feature volume to determine feature accessibility is that the whole feature volume, which has to be removed for creating a feature, should be accessible to the tool. Figures 7 and 8 show the global accessibility of a feature along two directions. For the direction in figure 7, the volume of intersection is NULL and hence the feature is globally accessible. For the direction in figure 8 , it is not accessible, as a finite volume is returned. In both the figures, the first block shows the feature volume on the part and the second block shows the extrusion of the volume along the direction of access. The subsequent frames show the intersection process (Aditya Narayan 1995) between the extrusion (considered one face at a time and shown in boldface) and the part. The last frame shows the result of the intersection. 


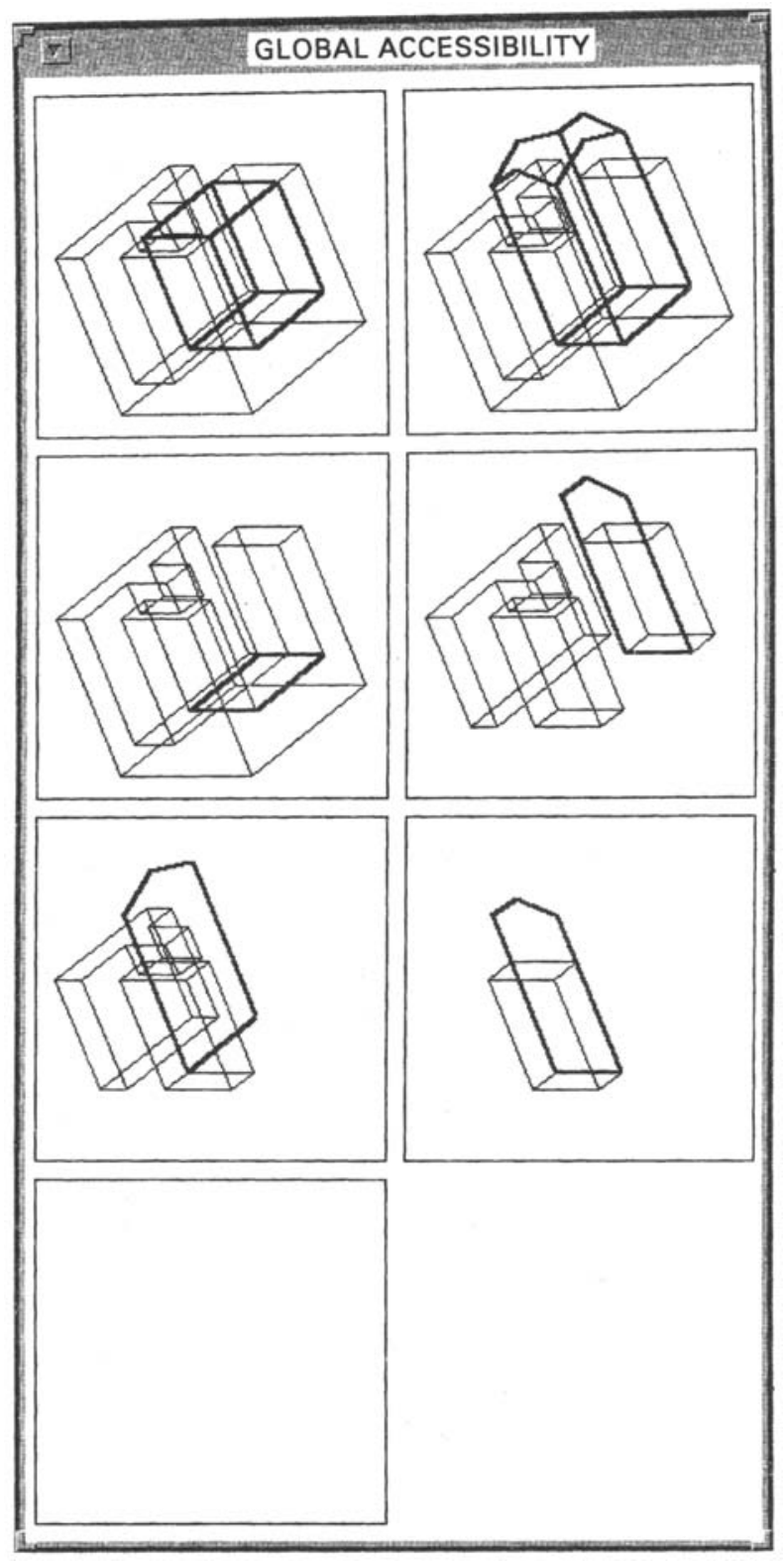

Figure 7. Globally accessible feature. 


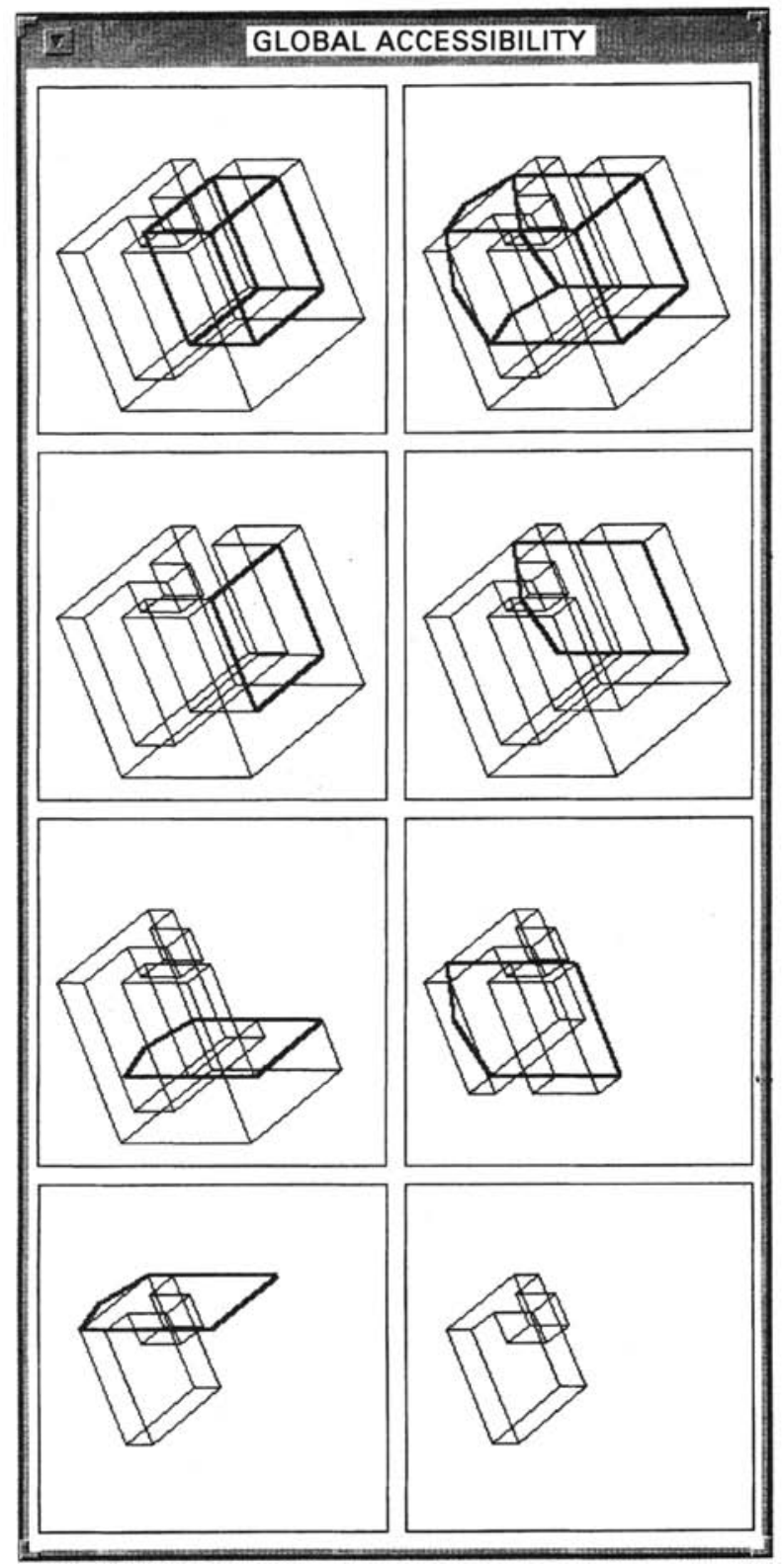

Figure 8. Globally inaccessible feature. 


\section{Accessibility and process planning}

Information about global accessibility of features in a part can be used to sequence the features for machining. If a feature is globally accessible with respect to the part, then that feature can be created in the stock independent of other features (figure 9). Also, if a feature is not globally accessible then that feature can be created only after the creation of a feature from whose volume it is accessible. In figure 10, features B and C are globally inaccessible features, whereas A is globally accessible. Features B and C can be created only if they are accessible with respect to a volume which has already been created. Once this precondition is satisfied they can be created in any sequence. Such features can be said to have secondary accessibility as they are dependent on creation of other features for

Part (a)

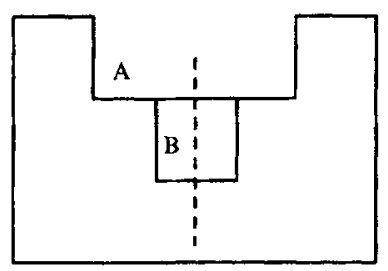

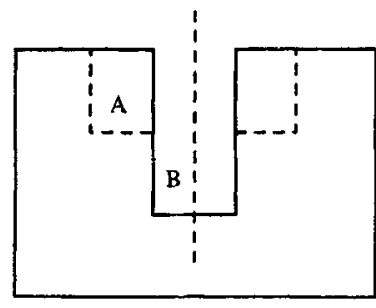

Interpretation 1

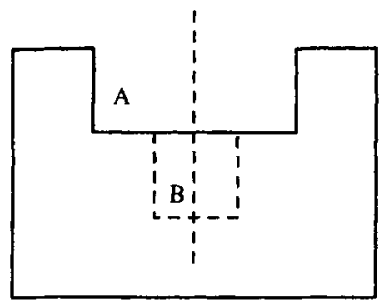

Interpretation 2

Part (b)

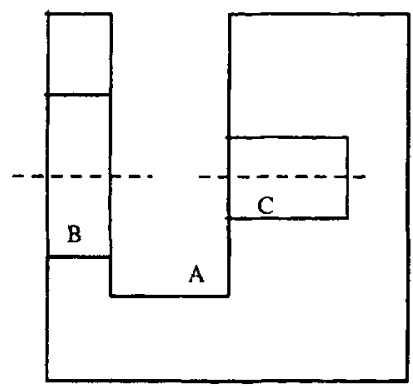

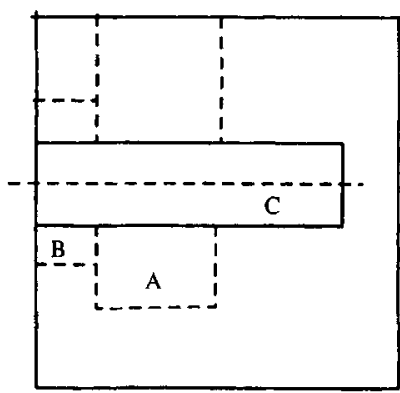

Interpretation 1

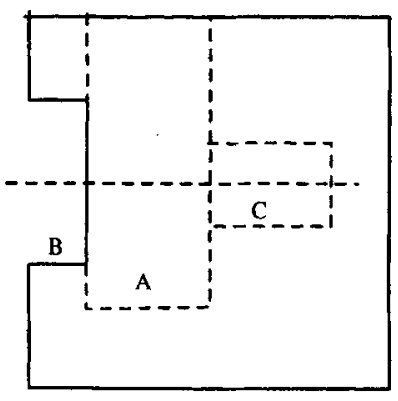

Interpretation 2

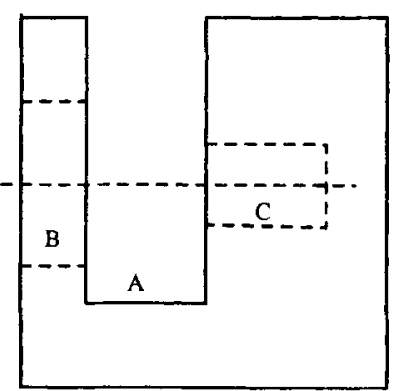

Interpretation 3

Figure 9. Machining sequence interpretations of globally accessible features. 


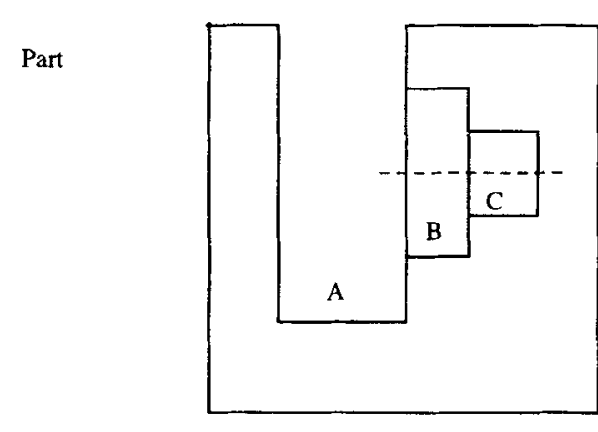

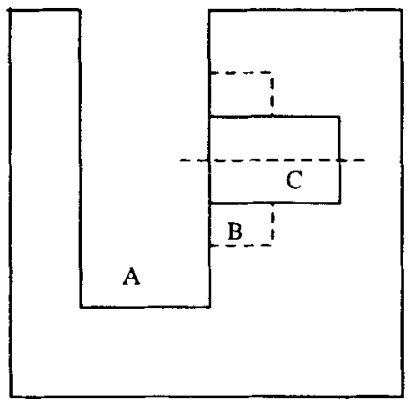

Interpretation 1

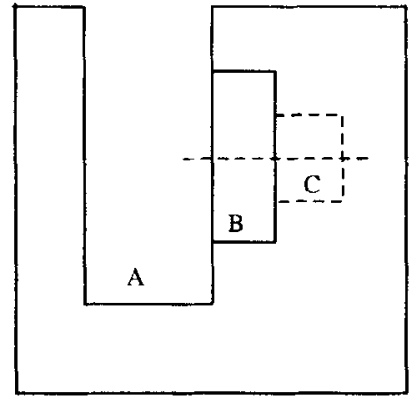

Interpretation 2

Figure 10. Machining sequence interpretations of globally inaccessible features.

becoming accessible. This dependency is not known a priori but has to be checked for the non-globally accessible features after the creation of every feature.

These assertions are based only on the consideration of spatial interrelationships between features and no other geometric or machinability-related constraints are operative. Based on the above, a tree of machining sequences, based on accessibility, can be generated where each path from the root to a leaf is a possible machining sequence from the accessibility point of view. At the root of the tree is the stock and at each leaf will be the final part if the part is manufacturable. At every level in the sequence tree, a feature will be created. The features at the first level are all globally accessible features as they can be created in the stock directly. At the other levels, the nodes will be the globally accessible features that have not been created at any node from the root to that node and features that gain secondary accessibility by the creation of features at ancestor nodes. An enumerated sequence tree is shown in figure 11 for a part with three features that are globally accessible and one feature that is globally inaccessible/exhibits secondary accessibility.

Features with secondary accessibility can be manufactured only if a tool-machine combination can be found that will reach it by fitting into an already created feature. These checks can be built into a tool selection module as constraints. This tree structure gives an exhaustive enumeration of all the possible machining sequences based on global accessibility. This allows the planner to consider all possible sequences in the search for an optimal process plan. There have been similar efforts reported (Nau et al 1992; Gupta \& Nau 1993), that use similar volume-based checks. However, in these efforts, the features 


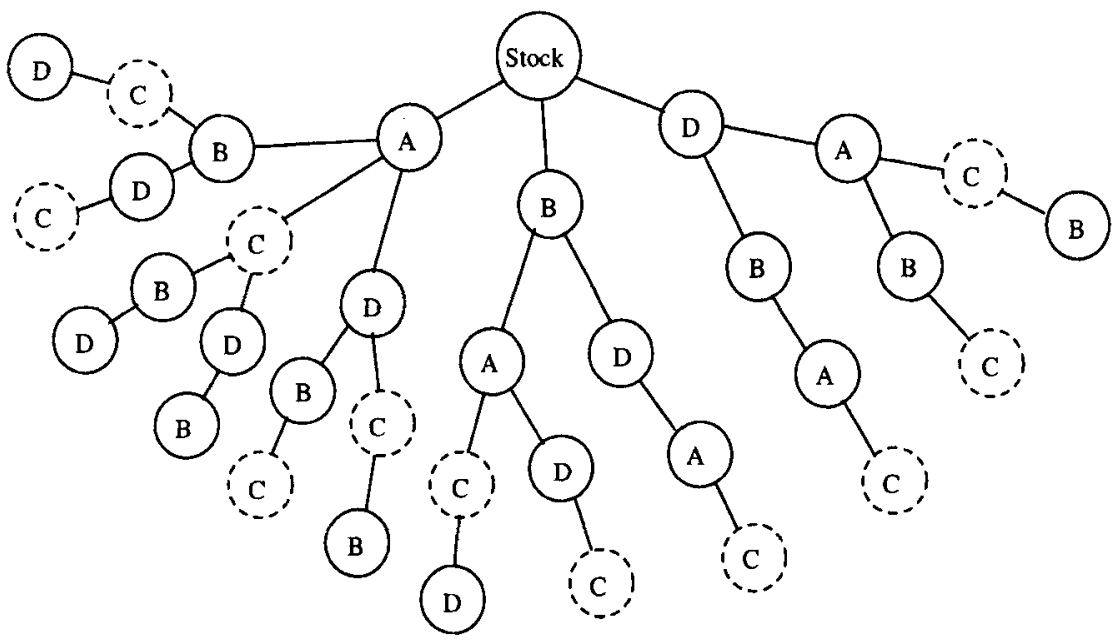

PART
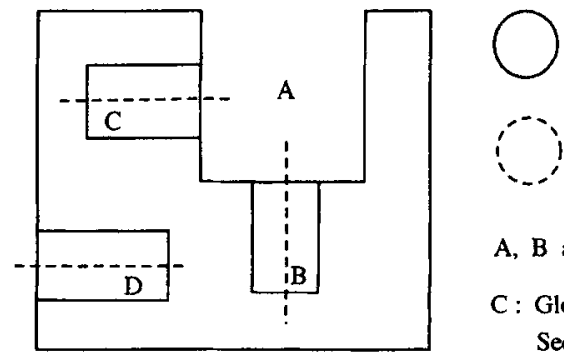

Globally Accessible Features

Secondary Accessibility Features

A, B and D: Globally Accessible Features

C : Globally Inaccessible/

Secondary Accessibility Feature

Figure 11. Enumerated sequence tree for a sample part.

and the volumes associated with them are pre-defined. Feasible machining sequences can be generated from the tree by the application of machining domain constraints such as dimensions, set-up minimisation and machining optimization, to mention a few.

The authors have not encountered any earlier attempts at interpreting the dimensions as constraints on machining sequence during generation of process plans. An attempt has been made to incorporate the dimensional information associated with the part design into process planning approaches (Aditya Narayan 1995).

The effect of specifying dimensional relationships between various entities in the part, such as abstract data, faces and edges, are mapped to features. The features are sequenced for machining based on these relationships. In the present implementation, only conventional linear and angular dimensions are handled. The dimension is assumed to be specified between two entities of the part, either abstract (e.g. centerline of a hole) or real (e.g. face, edge). This is a bidirectional relationship in which either entity may be referenced from the other. No directionality of reference is assumed, though a feature or face may be 'forced' to be a reference face by not specifying another entity with it in the dimension structure. The machining sequence that is generated based on the dimensional data in figure 12 is shown in figures 13 and 14. 


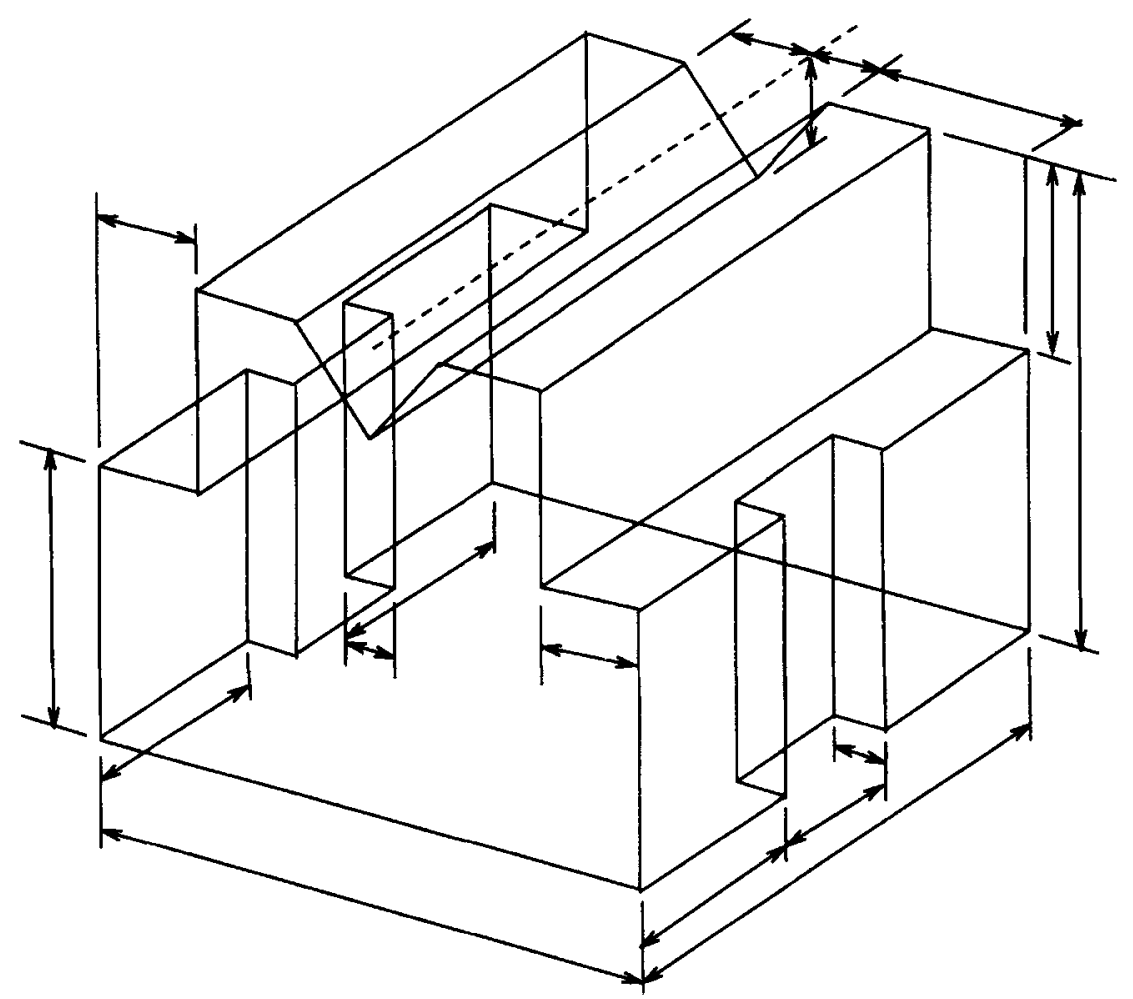

Figure 12. Test part with dimensions.

The sequence of machining based on dimensional data fulfills only the mandatory dimensional conditions specified by the part design. Further optimization of the process may be carried out by considering the freedom offered in set-up generation, tool change, cost minimisation etc. Also, constraints on the process will be imposed by tool and machine tool capability, their availability and fixturing. This can be done within the framework of the global accessibility tree by evaluating the various sequences that are enumerated by the tree.

Once the machining features have been sequenced, the volumes corresponding to the features can be found (Rao Nalluri et al 1995). Figure 13 shows the machining volumes corresponding to the creation of faces and features in each step of the sequence. Machining features provide the logic for selection and sequencing of operations. Machining volumes provide the geometric information for generating NC tool paths. The NC path generation module in figure 1 reasons over the machining volumes and generates the tool path (cutter location file) (Gurumoorthy 1996). In essence, once a component is modelled in the prototype system, $\mathrm{NC}$ tool paths required to realise the part from a given stock are automatically generated.

\section{Concluding remarks}

We have presented a framework based on features technology to support manufacturability evaluation and process planning. A prototype system as shown in figures 1 and 2 is under 


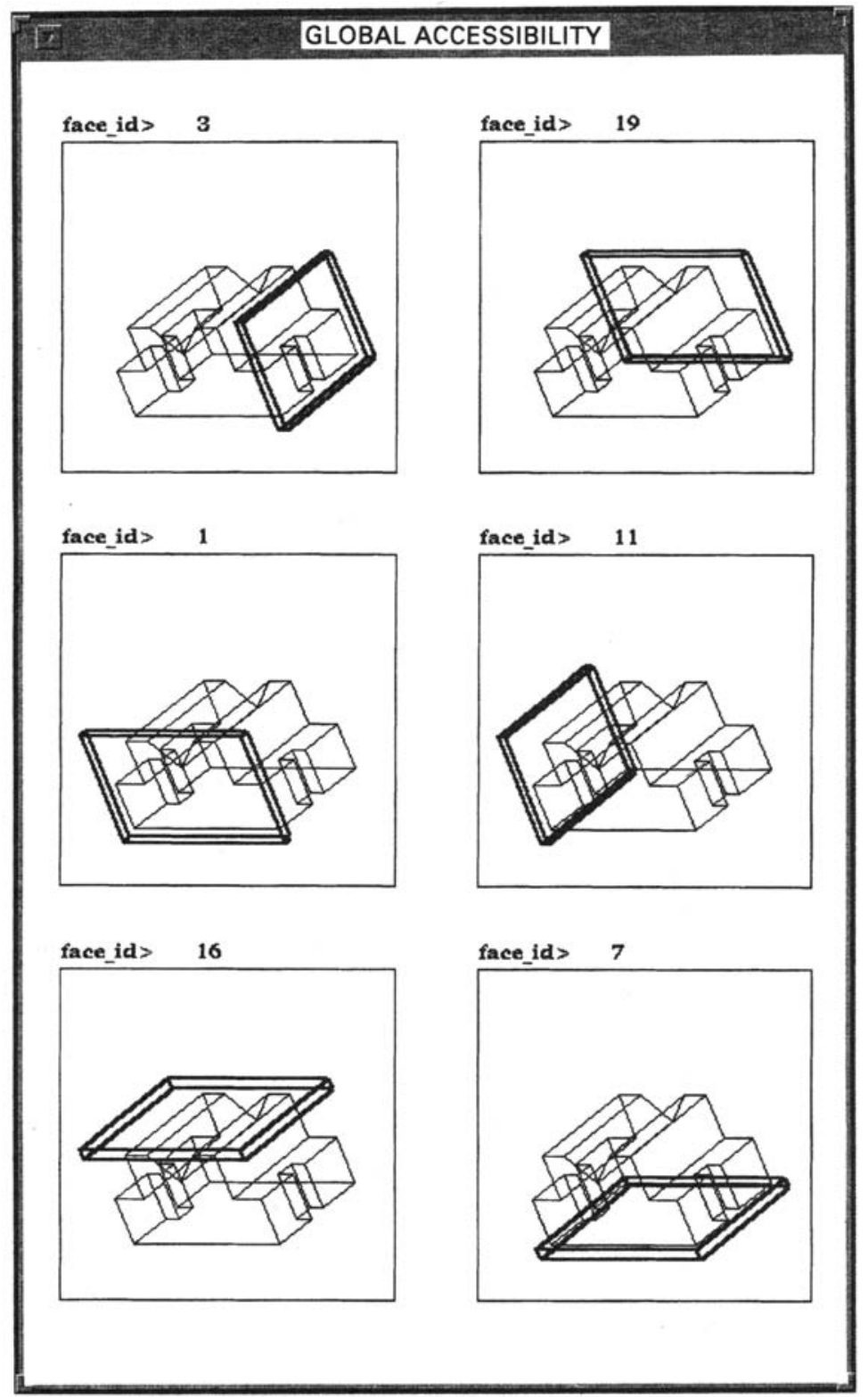

Figure 13. Machining sequence. 


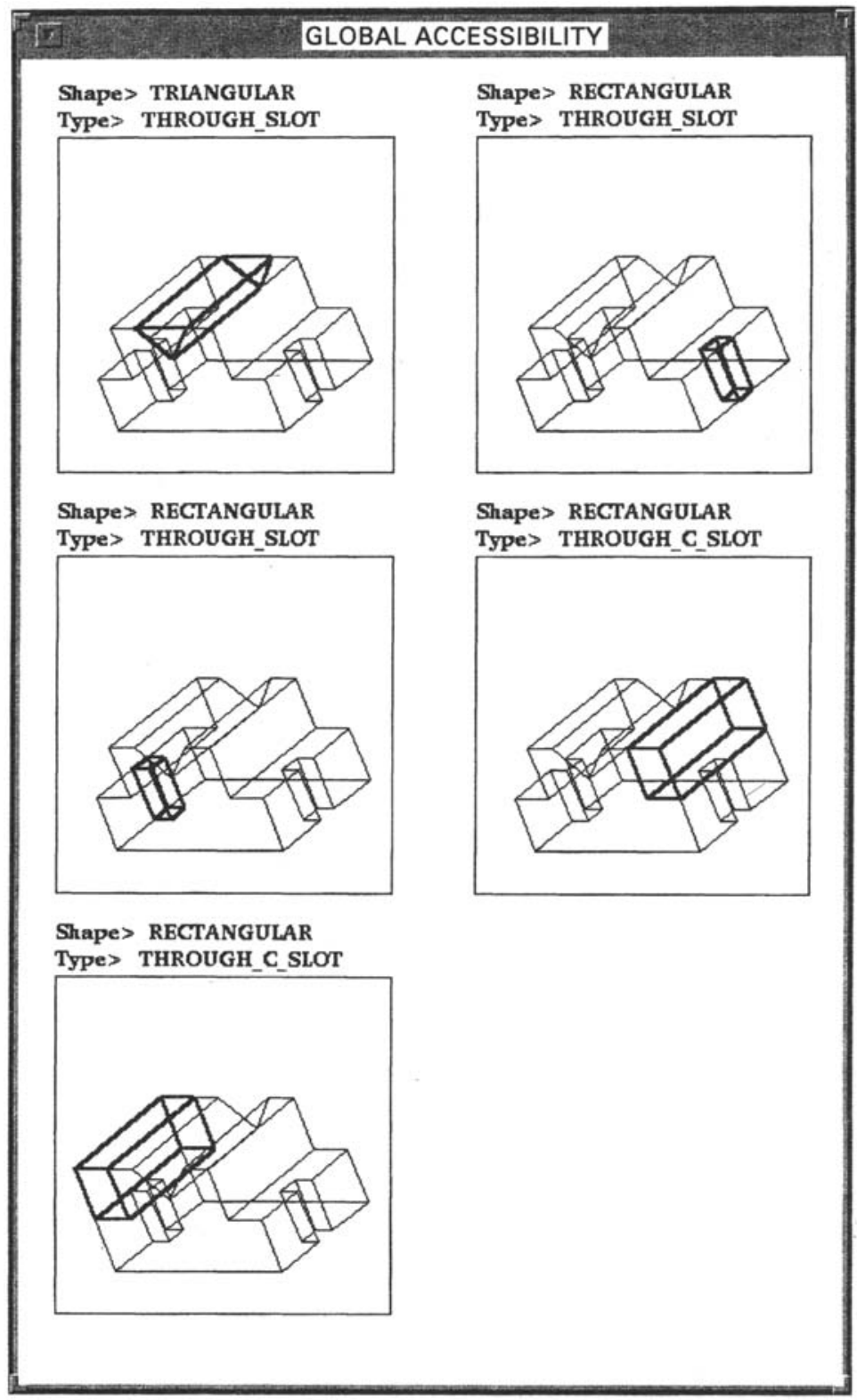

Figure 14. Machining sequence (contd.). 
development. Feature-based modelling based on direct editing, feature extraction, feature mapping and generation of sequence tree based on global accessibility information and automatic tool path generation have already been implemented for polyhedral solids. Direct editing of feature-based models and feature extraction have also been implemented for exact B-Rep models using the ACIS geometric kernel (Hari 1995; Gupta 1997). Currently the focus is on implementing constraints based on good machining practice (as followed on the shop floor) into both the evaluation and sequencing tasks.

In this paper, limitations and bottlenecks in the feature technology have been brought out and remedies have been outlined. Defining form features through feature generation model is a novel approach which has significant potential for features technology. Separation of 'type' and 'shape' reduces the effort involved in implementation of feature extraction and feature based modelling. The enumeration of DIF feature types gives a sound basis for design and implementation of feature-based systems. By removing (through updating) the recognised features, part geometry as seen by the feature extraction module progressively simplifies and eliminates problems in feature extraction (interacting/intersecting features) that have been reported in the literature. Use of features has been extended to geometric manipulation through development of algorithms for direct manipulation and editing of geometric representation. Manipulation of product data in mapping requires further investigation.

It has been shown that issues such as global accessibility of features are vital not only for the evaluation of designs for manufacturability but also have an effect on the sequencing of machining operations. Currently, the accessibility analysis considers only the spatial relationships between features. For process planning, the effect of feature sizes is important for the evaluation of machine and tool capability and for optimising machining volume removal. Also, a complete tool accessibility check would require evaluating the exact shape and size of the tool and the feature. Algorithms to determine local and global accessibility have to be extended to handle the more general case of features with free-form surfaces. The current implementation considers dimensional data and references for sequencing of machining operations. Further work could focus on issues such as form tolerances, setup minimisation, fixturing, tool and machine tool evaluation and machining practice and heuristics. The machining access directions determined based on the V_Map of features can be used for setup optimisation.

Global accessibility of features enable identifying multiple options in machining features. Work is underway to identify multiple feature interpretations across domains. For the integration of CAD geometric model with application domains to be completely automatic, redesign (i.e. modification of part geometry by applications) has to be possible. Some preliminary results are available in this area (Das et al 1996). The aim of the work presented in this paper has been to develop an algorithmic framework for process planning that is driven as much as possible by geometry and allows the incorporation of constraints and heuristics of the machining domain. It is hoped that further work in using DIF features as the unifying representation for geometric and domain specific reasoning will enable correlation of domain knowledge and constraints with part geometry to support concurrent product and process development. 
Work reported in this paper has been funded in part by grants from Aeronautical Development Agency and the Department of Science and Technology. Their financial support is gratefully acknowledged.

\section{References}

Aditya Narayan G 1995 Process planning framework for feature based manufacturing. Master of Eng. dissertation. Department of Mechanical Engineering, Indian Institute of Science, Bangalore

Arbab F 1992 Requirements and architecture of CAM oriented CAD systems for design and manufacture of mechanical parts. Ph D thesis, University of California at Los Angeles, Los Angeles, California

Butterfield W, Green M, Scott D, Stoker W 1986 Part features for process planning. CAM-I Report, R-86-ppp-01, November

Bakerjian R A (ed.) 1992 Design for manufacturability: Tool and manufacturing engineers handbook (Dearborn, MI: Soc. Manuf. Eng.) vol 6

CAD 1997 Computer-aided Design Report. CAD/CAM Publishing, San Diego, CA

Chang T C, Wysk R A 1985 An introduction to automated process planning systems (Englewood Cliffs, NJ: Prentice Hall)

Das D, Gupta S K, Nau D S 1996 Generating redesign suggestions to reduce setup cost: A step towards automated redesign. Comput. Aided Design 28: 763-782.

Gupta S K 1997 Feature-based editing of solid models, ME dissertation, Department of Mechanical Engineering, Indian Institute of Science, Bangalore

Gupta S K, Nau D S 1993 Generation of alternative feature-based models and precedence orderings for machining applications. In Proceedings of ACM Solid Modeling Conference, Montreal

Gurumoorthy B 1996 Reverse engineering of surfaces and solids and automatic NC path generation. Technical Report, TR/IISc/ME/CARL-P-96-3, Department of Mechanical Engineering, Indian Institute of Science, Bangalore

Hari S 1995 Feature extraction from exact B-Rep models. ME dissertation, Department of Mechanical Engineering, Indian Institute of Science, Bangalore

Joshi S, Chang T C 1988 Graph-based heuristics for recognition of machined features from 3D solid model. Comput. Aided Design 20(2): 58-66

Kyprianou L 1980 Shape classification in computer aided design. Ph D thesis, University of Cambridge, Cambridge, UK

Lim C P, Menq C H 1994 CMM feature accessibility and path generation. Int. J. Product. Res. 32: 597-618

Mill F G, Naish J C, Salmon J C 1994 Design for machining for a simultaneous engineering workstation. Comput. Aided Design 26: 521-527

Nau D S, Zhang G, Gupta S K 1992 Generation and evaluation of alternative operation sequences. Quality Assurance through Integration of Manufacturing Processes and Systems, ASME Winter Annual Meeting, pp 93-107

Rao Nalluri S R P 1994 Form features generation model for features technology. Ph D dissertation, Department of Mechanical Engineering. Indian Institute of Science, Bangalore

Rao Nalluri S R P, Gurumoorthy B 1993a Knowledge-based gluing operators for feature-based modelling, Comput. Ind. 23: 129-138

Rao Nalluri S R P, Gurumoorthy B 1993b Domain Independent Form Features for Concurrent Engineering. In Proceedings of JSME-ASME Joint Workshop on Design, Tokyo, pp 182-189 
Rao Nalluri S R P, Vani V, Gurumoorthy B 1995 A 3D clipping algorithm for form feature volume extraction. In Proceedings of International Conference on Computer Integrated Manufacturing, Singapore, pp 385-393

Sakurai H, Dave P 1996 Volume decomposition and feature recognition. Comput. Aided Design 27: 519-537

Salomons O W, van Houten F J A M, Kals H J J 1993 Review of research in feature-based design. J. Manuf. Syst. 12: 113-132

Shah J J 1988 Feature transformations between application specific feature spaces. Comput. Aided Eng. J. December: 247-255

Shah J J 1991 Assessment of features technology. Comput. Aided Design 23: 331-343

Shah J J, Shen Y, Shirur A 1994 Determination of machining volumes from extensible sets of design features. In Advances in feature based manufacturing (eds) J J Shah, M Mantyla, D Nau (New York: Elsevier Science) pp 129-157

Spyridi A J, Requicha A A G 1990 Accessibility analysis for the automatic inspection of parts. In Proceedings of IEEE International Conference on Robotics \& Automation, Cincinnati, pp 1284-1289

Subrahmanyam S, Wozny M 1995 Overview of automatic feature recognition techniques for computer-aided process planning. Comput. Ind. 26: 1-21

Tseng Y J, Joshi S B 1994 Recognizing multiple interpretations of interacting machining features. Comput. Aided Design 26: 667-688

Vandenbrande J H, Requicha A A G 1993 Spatial reasoning for the automatic recognition of machinable features in solid models. IEEE Trans. Pattern Anal. Mach. Intell. 15: 1269-1285

Woo T C, Chen L L 1992 Computational geometry on the sphere with application to automated machining. Trans. ASME J. Mech. Design 114: 288-295

Woo T C 1994 Visibility maps and spherical algorithms. Comput. Aided Design 26: 6-16 